\title{
Nuevo método estándar para la recolección selectiva de café
}

\section{New Standard Method for Picking Coffee ${ }^{1}$}

\author{
Ocampo-López Olga Lucía \\ Universidad Autónoma de Manizales, Colombia \\ Facultad de Ingeniería, Programa de Ingeniería Industrial \\ Correo: olocampo@autonoma.edu.co \\ Ovalle-Castiblanco Alex Mauricio \\ Universidad Autónoma de Manizales, Colombia \\ Facultad de Ingeniería, Programa de Ingeniería Industrial \\ Correo: movalle@autonoma.edu.co \\ Arroyave-Diaz Alejandro \\ Universidad Autónoma de Manizales, Colombia \\ Facultad de Ingeniería, Programa de Ingeniería Industrial \\ Correo: aarroyave@autonoma.edu.co
}

\author{
Salazar-Ospina Katherine \\ Universidad Autónoma de Manizales, Colombia \\ Facultad de Ingeniería, Programa de Ingeniería Industrial \\ Correo: ksalazar@autonoma.edu.co \\ Ramírez-Gómez Cesar Augusto \\ Centro Nacional de Investigaciones de Café, Colombia \\ Correo: cesara.ramirez@cafedecolombia.com \\ Oliveros-Tascon Carlos Eugenio \\ Centro Nacional de Investigaciones de café, Colombia \\ Correo: carlos.oliveros@cafedecolombia.com
}

\section{Resumen}

El café Colombiano es uno de los más suaves y de mejor calidad a nivel mundial por las variedades cultivadas, las condiciones ambientales, la recolección manual selectiva, el proceso de beneficio e industrialización. La herramienta utilizada en el método tradicional de recolección consiste en un recipiente plástico sujeto a la cintura del recolector. El Centro de Investigaciones del Café (CENICAFE) desarrolló el Canguaro 2M, un dispositivo novedoso para asistir la recolección, que consta de dos mangas unidas a un morral sujeto a la cintura y a los hombros del recolector. Este artículo presenta el método estándar de recolección con esta herramienta, definido por la aplicación de técnicas de ingenierías de métodos. Este nuevo método de recolección selectiva supone una serie de movimientos en el surco, en el árbol a través del surco, en el árbol y en la rama del cafeto; se identifican además los micromovimientos o therbligs de la recolección manual. El estudio incluye una comparación con el método tradicional y el análisis de indicadores de cosecha en términos de eficiencia, eficacia, calidad, y pérdidas. Los resultados permiten concluir que la adopción del nuevo método estándar para la recolección selectiva de café conlleva a un mejor desempeño en los indicadores de eficacia, calidad y pérdidas, en relación con el método tradicional, sin cambios significativos en la eficiencia del proceso.

Descriptores: método estándar, indicadores de cosecha, ingeniería de métodos, recolección de café, Therbligs.

\begin{abstract}
Colombian coffee is one of the mildest, high quality coffees in the world; this quality is owed to seeded varieties, environmental conditions, the selective manual harvesting, the postharvest and the industrial process. The tool used for harvesting traditional method is a plastic basket attached to the waist of the collector. The Center for Coffee Research (CENICAFE) developed the Canguaro 2M - a novel device that assists in the harvesting process, which consists of two sleeves joined to the backpack fastened to the waist and shoulders of the collector. This article presents a standard method of selective harvesting, which uses this tool and was defined applying the method engineering technique. This new method assumes a series of movements in the row, in the tree through the row, in the tree and in the branch of the coffee tree; the micro-movements or therbligs in manual harvesting are also identified. The study includes a comparison with a traditional method and analysis of harvesting indicators in terms of efficiency, efficacy, quality and losses. The results suggest that adoption of the new standard harvesting method using Canguaro 2M leads to a better performance in the harvesting indicators of efficacy, quality and losses, with respect to the traditional method, without significant changes in the process efficiency.
\end{abstract}

Keywords: standard method, harvesting indicators, method engineering, coffee picking, Therbligs.

\footnotetext{
${ }^{1}$ Este artículo es resultado del proyecto de Investigación titulado: “Análisis de métodos de recolección de café con el uso del Canguaro $2 \mathrm{M}$ y su impacto en la productividad", Convocatoria 566 Jóvenes Investigadores, financiado por COLCIENCIAS, la Universidad Autónoma de Manizales y CENICAFÉ.
} 


\section{INTRODUCCIÓN}

El café, una de las bebidas más populares a nivel global, es uno de los commodities más negociados en el mundo (González y Gutiérrez, 2012; Murthy y Naidu, 2012; Quintero y Rosales, 2014). Se cultiva en más de 70 países, la mayoría con pequeñas plantaciones menores a 10 hectáreas (Bitzer et al., 2009); se estima por tanto, que alrededor de 25 millones de pequeños caficultores producen $80 \%$ del café que se comercializa globalmente (Fairtrade Foundation, 2012). La industria cafetera es de gran relevancia en la economía mundial (Organización Internacional del Café, 2015, 2016) y fundamental en países en vías de desarrollo, como Colombia (Federación Nacional de Cafeteros, 2016).

Diferentes factores afectan el potencial de producción de un cultivo de café (Arcila et al., 2007; Poveda et al., 2014), por tanto, se requiere la aplicación de buenas prácticas (Turbay et al., 2014), en especial en las etapas de cosecha y beneficio que influyen en la calidad del producto final (FNC-CENICAFÉ, 2013). Para cosechar café se emplean técnicas como el desgranado en forma individual (picking) o masivo (stripping); en la primera, se toman principalmente los frutos maduros, dejando los demás para un pase de recolección posterior; en la segunda, se desprenden los frutos en todos los estados de desarrollo; al no ser selectiva, se obtiene un café con calidad diferente al anterior, con menor precio en el mercado internacional (Isaza et al., 2006; Farah, 2012).

En Colombia, la recolección manual selectiva es la técnica empleada para garantizar la calidad de los cafés suaves colombianos; sin embargo, incide en los costos del proceso, con una participación alrededor de $40 \%$ (Oliveros y Sanz, 2011).

Ante la necesidad e importancia de incrementar la productividad de la mano de obra en el proceso de recolección, CENICAFE ha realizado diferentes estudios que han permitido conocer los tiempos y movimientos empleados en proceso tradicional de recolección de café en Colombia (Vélez et al., 1999; Salazar et al., 2016) y el efecto de factores como la altura de la plantación (Villegas et al., 2005) y la pendiente del terreno (Martínez et al., 2005). Por otra parte, se han aplicado técnicas electrónicas para la identificación de frutos maduros, con el fin de lograr la cosecha selectiva (Cadena, 2011); se han evaluados diferentes técnicas, equipos y herramientas para la cosecha de café (López et al., 2008a; 2008b; Oliveros y Sanz, 2011) y se ha avanzado en la identificación geométrica del fruto y las frecuencias naturales de vibración (Tinoco et al., 2014).
Entre los dispositivos desarrollados para mejorar los indicadores de la recolección está el Canguaro 2M (Ramírez et al., 2011; 2013), que permite eliminar movimientos innecesarios (Arroyave et al., 2014) que se presentan con el sistema tradicional (Salazar et al., 2016). Para facilitar su uso y adopción por los recolectores, se requiere realizar estudios que permitan definir un método estándar, con el cual se obtengan las ventajas esperadas frente al sistema tradicional. Las variables asociadas a los indicadores de cosecha, eficiencia, eficacia, calidad y pérdidas, pueden emplerse para el análisis de esta herramienta (Salazar et al., 2014).

La ingeniería de métodos establece procedimientos sistemáticos en las operaciones, facilitando su mejoramiento y optimización (Niebel y Freivalds, 2004); su aplicación en la agroindustria es cada vez mayor (Spinelli y Visser, 2009; Torres et al., 2010; Carreira et al., 2013). Utiliza técnicas para el análisis de operaciones como dividir una tarea en elementos de trabajo y estudiar cada movimiento para ordenarlo o eliminar los innecesarios (Niebel y Freivalds, 2004). Comprende dos fases de estudio, la primera abarca el análisis visual de los macro y micromovimientos en los cuales deben considerarse los principios de la economía de movimientos; en la segunda se define el tiempo requerido para efectuar la tarea según una norma de ejecución preestablecida (Durán, 2007; Kanawaty, 2011). El uso de dispositivos, como las cámaras digitales y software especializados para el análisis de los datos, buscan determinar una mayor confiabilidad en los datos, eliminando la subjetividad del observador y minimizando el error humano en la captura de información cuando se utiliza la observación directa (Tinoco et al., 2015; Ovalle y Cardenas, 2016).

El estudio de los micromovimientos o therbligs se realiza en trabajos de mucha actividad y repetición (Meyers, 2000), donde vale la pena examinar con mayor detalle y determinar dónde es posible ahorrar movimientos de manera que el operario pueda realizarlos con el mínimo de esfuerzo y fatiga (Kanawaty, 2011).

Este artículo presenta un estudio de los macro y micro movimientos del proceso de recolección del fruto de café para evaluar los indicadores de la cosecha y los impactos en la productividad de la mano de obra y la calidad. Los resultados de este estudio determinan la definición de un nuevo método estándar para asistir la recolección de café con el dispositivo Canguaro 2M, que se presenta en este trabajo.

\section{Desarrollo}

Herramienta de ReColección- Canguaro 2M 
El Canguaro 2M es un dispositivo, diseñado por CENICAFÉ (Ramírez et al., 2011), para la cosecha manual de café; está conformado por dos mangas que se fijan a las manos del recolector mediante resortes a manera de guante abierto; la manga dispone de un aro plástico para facilitar su apertura y el ingreso de los frutos desprendidos, que descienden hasta un bolso sujeto a la cintura y hombros del recolector. Está fabricado en tela impermeable y tiene una capacidad de $12 \mathrm{~kg}$. La figura 1 presenta algunas fotografías que ilustran esta herramienta.

\section{HeRRAMIENTA DE RECOLECCIÓN TRADICIONAL}

El sistema tradicional es comúnmente empleado en Colombia; consiste en un recipiente plástico, con una capacidad de 10 a 12 kilogramos, sujeto a la cintura del recolector por medio de una banda, como se ilustra en la figura 1.

\section{MÉTODOS Y PRUEBAS REALIZADAS}

A través de la ingeniería de métodos se establecieron las características especiales de cada una de las operaciones evaluadas en el proceso de recolección de café, y se identificaron los macro y micro movimientos en la ejecución de la tarea; se analizaron los principios de economía de movimientos (Kanawaty, 2011) en especial los movimientos simétricos y simultáneos realizados con las manos, y el trabajo en la zona de confort o área máxima de alcance de las manos.

\section{RECOLECCIÓN DE INFORMACIÓN}
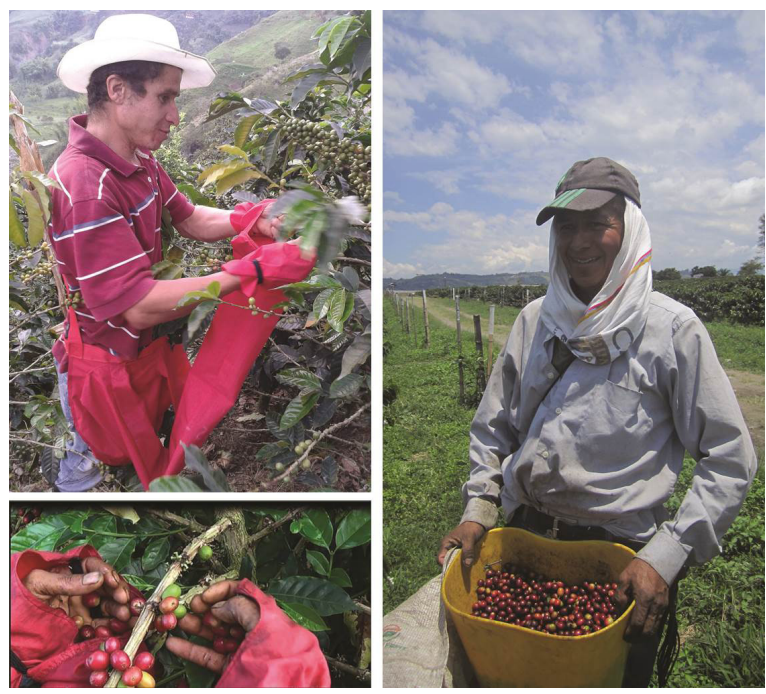

Figura 1. Recolección con Canguaro 2M (izquierda) y método tradicional (derecha)
Los protocolos de seguimiento y medición se definieron en pruebas piloto en campo, en fincas cafeteras del municipio de Manizales (Caldas, Colombia). El estudio se realizó en dos plantaciones, la primera localizada en la Vereda Naranjal, municipio de Chinchiná (Caldas, Colombia) y otra en el municipio de Cajibío (Cauca, Colombia), durante los inicios y fines de cosecha 20132014. La primera plantación se caracterizó por ser variedad Castillo, de segunda y cuarta cosecha; sembrada en lotes de 1 ha; con densidades de siembra entre 5.000 y 10.000 plantas por ha. La segunda, estaba sembrada en lotes de 1 ha, con variedades Caturra, Borbón, Típica y Castillo, con densidades de siembra en promedio de 5000 árboles por ha.

La unidad de análisis fue el recolector, para su selección se consideró el nivel de experiencia mayor a un año, tanto con el método tradicional como con el Canguaro 2M. La muestra consideró 23 recolectores localizados en las zonas de estudio, que cumplieron con los criterios de inclusión definidos. Cada recolector firmó el consentimiento informado para la participación en la investigación. CENICAFÉ reportó que el número de recolectores que ha adoptado el Canguaro 2M, ascendió a 314 para el año 2012 (Ramírez et al., 2013).

Para el seguimiento en campo se empleó la observación directa con registro en planillas y el método cinematográfico o uso de video que consiste en filmar al trabajador con una cámara a una velocidad determinada y después analizar la filmación. Este seguimiento se realizó a cada recolector por espacio de una hora, durante la recolección, iniciando con el método tradicional y después, con el Canguaro 2M.

Durante el seguimiento se registraron los macromovimientos del recolector en el surco, en el árbol a través del surco, en el árbol y en las ramas del cafeto durante la recolección de café. Se tomaron filmaciones de los micromovimientos en la recolección selectiva del fruto, desde el desprendimiento del fruto hasta el almacenamiento temporal en la herramienta de recolección. Los registros se efectuaron con una cámara Sony Handycam PJ30, con una calidad de imagen HD y una resolución de 60 cuadros por segundo.

\section{INDICADORES DE COSECHA}

Este estudio consideró el desempeño de la herramienta de recolección a través de la medición de los indicadores de cosecha que se ilustran en la figura 2. Su análisis implicó las siguientes mediciones en campo para cada recolector y con cada método empleado: 
- El indicador de calidad se obtuvo por el porcentaje que representa el peso de frutos maduros en una muestra de un kilogramo de café cosechado.

- El indicador de eficiencia se estimó como los kilogramos de café cosechados por hora transcurrida.

- El indicador de eficacia se calculó como el número de frutos maduros no cosechados por árbol; este dato se registró por observación y conteo directo después de la recolección, por cada árbol.

- El indicador de pérdidas se estimó por el número de frutos caídos al suelo por árbol, este dato también se registró por observación y conteo directo, por cada árbol cosechado.

\section{ANÁLISIS DE INFORMACIÓN}

Mediante el software libre Camtasia studio 8 se realizó la desfragmentación de las imágenes de video en fotografías. Se utilizó el programa Free video to jpg converter para la descomposición del video en una serie de fotografías a 0.016 segundos.

El software Microsoft Excel se empleó para la organización de los registros físicos y el análisis gráfico de la información. Las pruebas estadísticas se realizaron con el software IBM SSPS Statistics.

El análisis de frecuencia permitió identificar los movimientos realizados por los recolectores, que se compararon con los principios de economía de movimientos para identificar el método estándar.

El análisis de varianza ANOVA con la prueba post hoc de Duncan y el test de Kruskal Wallis permitieron identificar diferencias significativas $(\alpha=0.05)$ en los indicadores de cosecha entre los recolectores. Por otra parte, se emplearon pruebas de comparación de medias como la prueba $t$ y la $U$ de Mann Whitney para establecer diferencias $(\alpha=0.05)$ entre los métodos de recolección.

\section{Discusión y ANÁlisis DE RESULTADOS}

\section{Movimientos en el PROCESO DE RECOLECCIÓN}

Durante el proceso de recolección tanto con Canguaro $2 \mathrm{M}$ como con el dispositivo tradicional, es posible identificar los siguientes movimientos que se describen a continuación:

- Movimientos en el surco: Se identificaron dos métodos para realizarlo; en el primero los recolectores inician cerca al punto de pesaje; mientras que en el segundo realizan un desplazamiento hasta el surco más lejano de la caseta de pesaje para iniciar la recolección. Según el seguimiento realizado, el segundo método se empleó por $70 \%$ de los recolectores, tanto en terrenos planos como con pendiente.

En el estudio de movimientos previo realizado por Velez et al. (1999) para el método tradicional se identificaron 5 trayectorias para realizar estos desplazamientos en el surco, 3 de ellas en terrenos pendientes denominadas trayectorias A, B y C, y 2 para terrenos planos, D y E. La trayectoria A es equivalente al primer método; la trayectoria B es el segundo método identificado en este estudio; la trayectoria $\mathrm{C}$ que corresponde a una recolección paralela al sentido de la pendiente no se logró identificar en esta investigación; la trayectoria $\mathrm{D}$ equivale al primer método para terreno plano y finalmente, la trayectoria $\mathrm{E}$ es el segundo método para terrenos planos. En consecuencia, se confirma que solo se evidencian dos métodos para los movimientos, según el tipo de terreno.

- Movimiento en el árbol a través del surco: En este caso se identificaron tres tipos de movimientos: circular o "rolos", por caras y en serpentín como se ilustran en la figura 3. En el primero se recogen los frutos del árbol en un movimiento circular o una vuelta com-

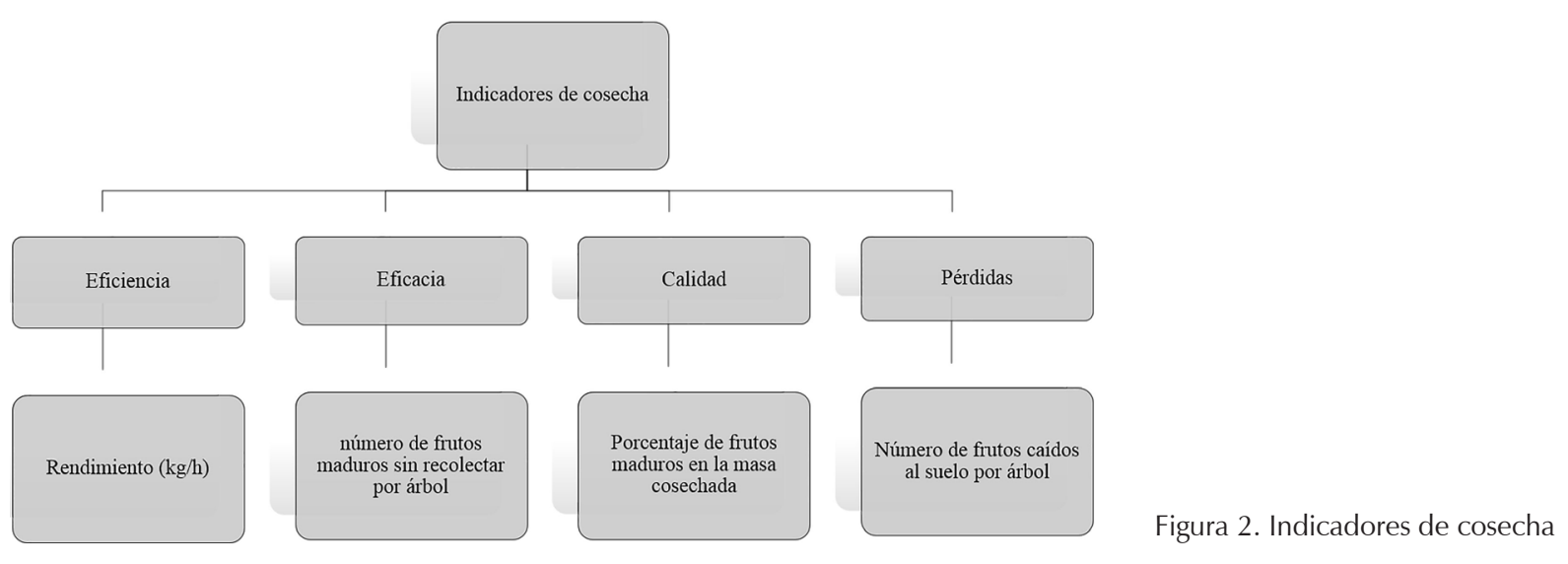


pleta. En el segundo se realiza el desprendimiento de los frutos por una sola cara o lado del árbol hasta terminar el surco asignado. En el tercero se desprenden los frutos de las caras laterales realizando un movimiento en zigzag en el surco y su posterior retorno.

El método circular se empleó por $100 \%$ de los recolectores durante el seguimiento; los demás se reportaron en entrevista; según los recolectores, este método permite una mejor recolección en una sola vuelta al árbol. El análisis de economía de movimiento concluye además que es el más efectivo en la recolección. Pero trae consecuencias como el desprendimiento involuntario con el cuerpo y con el recipiente plástico de frutos en los árboles vecinos o contiguos.

- Movimientos en el árbol: La figura 4 sintetiza los movimientos identificados en el árbol. En el método 1 , el recolector realiza un desprendimiento de los frutos desde la copa hasta la base del árbol, se presenta cuando los árboles son de porte alto. El método 2 es inverso al anterior y se presenta cuando los árboles son de porte bajo. En el tercer método, el recolector empieza por la parte central del árbol, continuando hacia la copa, regresa al centro y termina en la base, aprovechando esta posición para recolectar los frutos que están en el suelo. El cuarto método es inverso al anterior.

Se evidencia que el tercer método es el más frecuente tanto en la recolección con Canguaro $2 \mathrm{M}$ como con el sistema tradicional (figura 5); debido a que le permite al recolector estar más en la zona central o de confort, como lo indican los principios de economía de movimientos (Kanawaty, 2011).

- Movimientos en las ramas: En este caso se identificaron cuatro métodos que se esquematizan en la figura

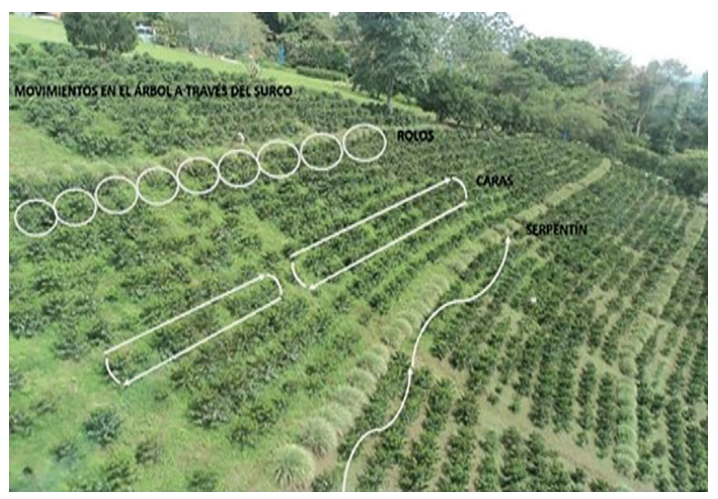

Figura 3. Movimientos en el árbol a través del surco
6. Para el método 1, el recolector realiza el desprendimiento de los frutos desde el tallo hacia la punta de la rama; con un trabajo simultáneo de las manos. En el método 2, la recolección de los frutos se efectúa desde la punta de la rama hasta el tallo. Para el método 3 se tienen dos etapas, la primera de la mitad de la rama hacia la punta y la segunda del tallo hacia la mitad; este método se utiliza en ramas largas con alta carga de frutos. En el método 4, el recolector adopta una posición perpendicular a la rama para el desprendimiento de los frutos.

En el seguimiento realizado, los recolectores emplearon con una mayor frecuencia el método 1 , como se ilustra en la figura 7, tanto en la recolección con Canguaro como con el sistema tradicional. Este método utiliza el principio de economía de movimientos que hace referencia al movimiento simétrico y al tiempo con las dos manos (Kanawaty, 2011). En este sentido, los movimientos con una trayectoria y sin cambio de dirección son los más eficientes.

\section{Micromovimientos en El PROCESO DE RECOlECCIÓN}

A partir del procesamiento de imágenes y basados en el estudio de Therbligs reportados por Gilberth, la identificación de los micromovimientos en el proceso de recolección con Canguaro 2M comprende: Buscar y seleccionar (B-Se) el fruto, alcanzarlo ( $\mathrm{Al}$ ), tomarlo (T), soltarlo a la palma (Sp), sostenerlo (So), mover (M) la mano con carga para buscar otro fruto, para finalmente soltar la carga (Sc) en el Canguaro 2M, según se esquematiza en la figura 8.

En este sentido, puede identificarse el ciclo básico de recolección que comprende los movimientos efectivos y el subciclo que abarca los movimientos innecesarios.

- Ciclo básico de recolección: Inicialmente, el recolector busca y selecciona (B-Se) el fruto; mueve las manos hasta alcanzar (Al) el fruto seleccionado; realiza el desprendimiento y toma (T) el fruto; suelta el fruto (Sc) dentro de la manga del Canguaro 2M como se ilustra en la figura 8.

- Subciclo de recolección: Comprende los movimientos soltar a la palma (Sp), sostener (So) y mover (M) como se visualiza en la figura 8.

Por otra parte, el seguimiento permitió identificar los micromovimientos con el método tradicional. Inicialmente, el recolector busca y selecciona (B-Se) el fruto maduro, lo alcanza (Al), toma o desprende el fruto de la rama $(\mathrm{T})$, suelta el fruto a la palma de la mano (Sp); lo 


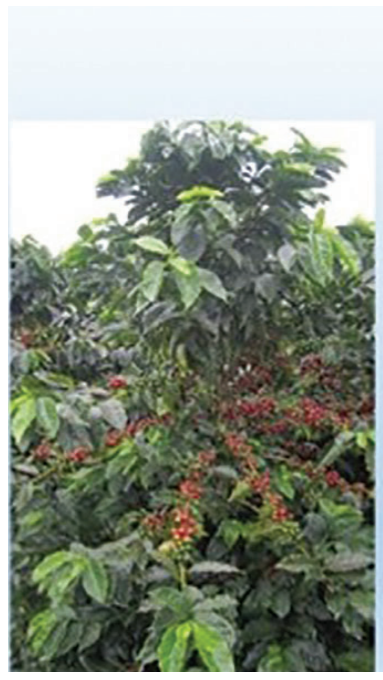

\section{Movimientos en el árbol}

\section{Método1}

Método2

Método3

Método4

1

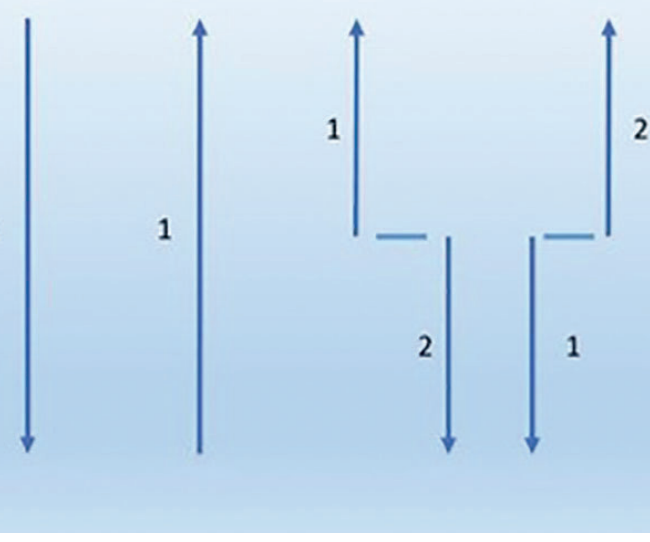

Figura 4. Movimientos en el árbol

Figura 5. Frecuencia de movimientos en el árbol con los sistemas de recolección evaluados

Figura 6. Movimientos en las ramas

Figura 7. Frecuencia de movimientos en las ramas con los sistemas de recolección evaluados 
sostiene en la mano (So), mueve las manos hacia el recipiente $(\mathrm{M})$ y suelta el fruto cosechado en el recipiente de almacenamiento (Sc). Este ciclo se esquematiza en la figura 8, coincide con lo reportado por estudios previos (Arroyave et al., 2014; Salazar et al., 2016).

Existe por tanto, una diferencia en el micro movimiento mover $(\mathrm{M})$ y los dos sistemas de recolección; en el tradicional hace parte del ciclo básico de recolección e implica el desplazamiento de las manos con carga hacia el recipiente plástico; con el Canguaro 2M, mover (M) implica un desplazamiento de la mano con carga en búsqueda de otro fruto para desprenderlo. En consecuencia, el Canguaro 2M presenta ventajas por la eliminación del micromovimiento que transporta los frutos almacenados en la mano hacia el recipiente.

Con el procesamiento de las imágenes de video se identificó el tiempo promedio para la recolección de un fruto de café, presentado en la tabla 1; se incluyen además los niveles de significancia de las pruebas de comparación de medias, que reflejan diferencias estadísticamente significativas ( $\alpha=0.05 \%$ ) en el tiempo promedio de recolección de un fruto de café entre los métodos de recolección manual; el menor valor se presenta con el Canguaro 2M en comparación con el sistema tradicional.

Por otra parte, la técnicas de descomposición de imágenes permitieron establecer los tiempos de cada Therbligs para la recolección con Canguaro 2M; el análisis estadístico de la información se resume en la tabla 2 que presenta los valores medios, el intervalo de confianza para la media a $95 \%$, el coeficiente de variación (CV) y la participación porcentual de cada Therblig en el ciclo de operación.

El análisis de varianza ANOVA permite identificar diferencias estadísticamente significativas ( $\alpha=95 \%$ ) entre los recolectores en los Therblighs alcanzar (significancia $0.040^{*}$ ) y soltar carga (significancia $0.00^{*}$ ). Por otra parte, la prueba no paramétrica de Kruskal Wallis evidencia diferencias entre los recolectores en el micromovimiento soltar-carga (significancia $0.021^{*}$ ).

\section{INDICADORES DEL PROCESO DE RECOLECCIÓN}

Los indicadores de cosecha permiten la evaluación operativa global del proceso de recolección de café en términos de eficiencia, eficacia, calidad y pérdidas. Estos indicadores se evaluaron en la recolección tanto con Canguaro $2 \mathrm{M}$ como con el método tradicional. Los resultados comparativos se presentan en la tabla 3, que resume la estadística descriptiva y la significancia (sig.) de las pruebas de comparación de medias. Se evidencian mejoras en los indicadores de eficacia, calidad y pérdidas con el Canguaro 2M sin diferencias significativas en el rendimiento.

Estos resultados evidencian el cumplimiento de los objetivos propuestos por CENICAFÉ durante el diseño del Canguaro 2M (Ramírez et al., 2011; 2013); sin embar-

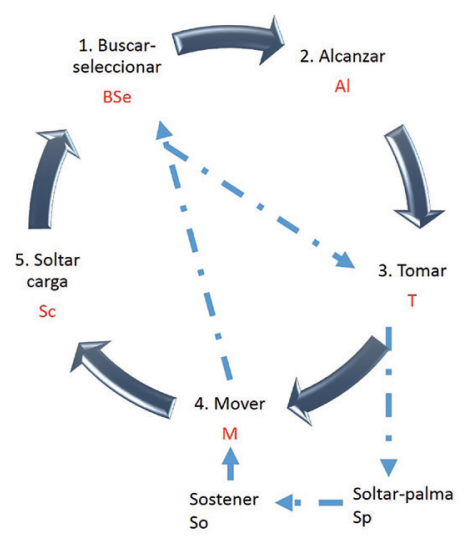

Método tradicional

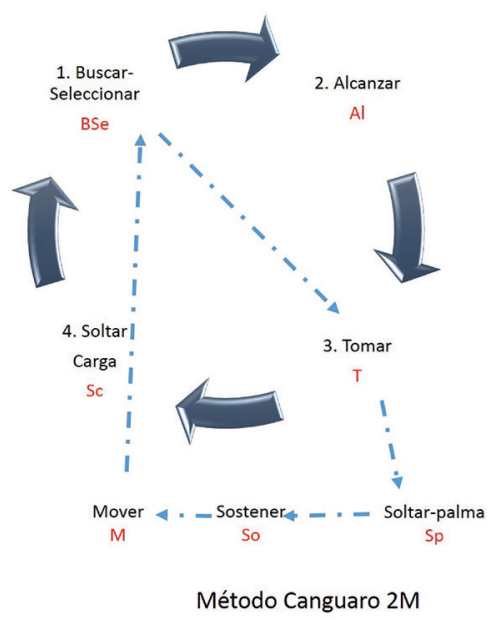

Figura 8. Therbligs para la recolección con el Canguaro $2 \mathrm{M}$ y el método tradicional

Tabla 1. Tiempo promedio de ciclo para recolectar un fruto según el método empleado

\begin{tabular}{cccccc}
\hline & \multicolumn{2}{c}{ Tiempo promedio para recolectar un grano } & \multicolumn{2}{c}{ Significancia } \\
\hline Método & $\begin{array}{c}\text { Tiempo promedio } \\
\text { ciclo (s) }\end{array}$ & Desviación típica & $\begin{array}{c}\text { Error típico de la } \\
\text { media }\end{array}$ & Prueba t & $\begin{array}{c}\text { Prueba U-Mann } \\
\text { Whitney }\end{array}$ \\
\hline Tradicional & 0.61 & 0.015 & 0.002 & $0.00^{*}$ & $0.00^{*}$ \\
Canguaro 2M & 0.52 & 0.023 & 0.003 & \\
\hline
\end{tabular}


Tabla 2. Tiempos por Therbligs con método Canguaro 2M

\begin{tabular}{|c|c|c|c|c|c|c|}
\hline \multirow[b]{2}{*}{ Therblig } & \multicolumn{3}{|c|}{ Estadística descriptiva } & \multicolumn{3}{|c|}{$\begin{array}{c}\text { Participación porcentual en el ciclo de } \\
\text { operación }\end{array}$} \\
\hline & $\begin{array}{c}\text { Tiempo } \\
\text { promedio } \\
\text { Therblig (s) }\end{array}$ & $\begin{array}{l}\text { Intervalo } \\
\text { confianza }\end{array}$ & $\mathrm{CV}$ & $\begin{array}{l}\text { Límite } \\
\text { superior }\end{array}$ & Media & Límite inferior \\
\hline Tomar & 0.082 & 0.002 & $7 \%$ & $15.90 \%$ & $15.77 \%$ & $15.64 \%$ \\
\hline Soltar a la palma & 0.064 & 0.001 & $4 \%$ & $12.52 \%$ & $12.31 \%$ & $12.10 \%$ \\
\hline Alcanzar & 0.103 & 0.004 & $13 \%$ & $19.68 \%$ & $19.81 \%$ & $19.93 \%$ \\
\hline Mover & 0.078 & 0.002 & $9 \%$ & $15.11 \%$ & $15.00 \%$ & $14.90 \%$ \\
\hline Sostener & 0.081 & 0.002 & $10 \%$ & $15.71 \%$ & $15.58 \%$ & $15.46 \%$ \\
\hline Soltar carga & 0.064 & 0.003 & $17 \%$ & $12.13 \%$ & $12.31 \%$ & $12.48 \%$ \\
\hline $\begin{array}{l}\text { Buscar- } \\
\text { seleccionar }\end{array}$ & 0.048 & 0.003 & $0 \%$ & $8.95 \%$ & $9.23 \%$ & $9.50 \%$ \\
\hline
\end{tabular}

go, se puede determinar que la mejora en el rendimiento aún no ha sido posible con esta herramienta de recolección Canguaro 2M. Una de las causas que afecta este rendimiento es la adopción de micromovimientos innecesarios relacionados con el almacenamiento de frutos en la mano.

\section{MÉTODO ESTÁNDAR PARA LA RECOLECCIÓN CON Canguaro $2 \mathrm{M}$}

Para la utilización adecuada de la herramienta Canguaro $2 \mathrm{M}$ se deben tener en cuenta los siguientes movimientos:

- Movimientos en el surco: Realizar el desplazamiento hasta el surco más lejano al punto de pesaje para iniciar la recolección.

- Movimientos en el árbol a través del surco: Para una recolección completa del árbol se sugiere el movimiento por "rolos" o de una forma circular.

- Movimientos en el árbol: Realizar este movimiento en dos etapas: la primera, desde la mitad del árbol hasta la copa del árbol y en la segunda, desde la mitad del árbol hacia la parte inferior; finalizar con la recolección de los frutos que se encuentran en el suelo.

- Movimientos en la rama: Adoptar una posición frontal para lograr un movimiento simultáneo con las dos manos y realizar la recolección desde el tallo hacia afuera.

- Micromovimientos de las manos: El recolector requiere de entrenamiento para que solo adopte los Therbligs del ciclo básico de recolección: Buscar-Seleccionar, alcanzar, tomar y soltar carga; en consecuencia, debe eliminar micromovimientos que impliquen el almacenamiento de frutos en la mano.

\section{Conclusiones}

Esta investigación da respuesta a las preguntas formuladas relacionadas con el método estándar para la reco- lección de café con el uso de Canguaro $2 \mathrm{M}$ y el comportamiento de los indicadores del proceso de recolección de café.

Los macromovimientos que se realizan en la recolección con Canguaro 2M no difieren del método tradicional. Se clasificaron en 4 tipos según los movimientos en el surco, en el árbol a través del surco, en los árboles y en las ramas. Se identificaron dos movimientos en el surco dependiendo de la pendiente del terreno; tres movimientos en el árbol a través del surco; cuatro movimientos en los árboles y cuatro movimientos en las ramas.

El ciclo básico de la recolección con Canguaro 2M comprende los micromovimientos o Therbligs: 1) Buscar-Seleccionar, 2) Alcanzar, 3) Tomar y 4) Soltar Carga. En comparación con el ciclo de recolección con el sistema tradicional, se concluye que el Canguaro 2M presenta una ventaja fundamental con la eliminación de los movimientos que corresponden al llevar los frutos almacenados en la mano hacia el recipiente.

Con la identificación de los macro y micro movimientos se estableció el método estándar de recolección con el Canguaro 2M que comprende el desplazamiento hasta el surco más lejano al punto de pesaje; la recolección del árbol en forma circular; los movimientos en el árbol en dos etapas, iniciando en la parte central del dosel hasta la copa y luego desde la mitad hacia la parte inferior; el desprendimiento de los frutos de la rama iniciando desde el tallo hasta la punta, y la ejecución del ciclo básico de recolección.

Este estudio concluye que el uso del Canguaro 2M trae beneficios en los indicadores de cosecha, eficacia y pérdidas en relación con el método tradicional, sin cambios representativos en el indicador de eficiencia. Sin embargo, el análisis de los micromovimientos refleja que es posible mejorar la eficiencia con el Canguaro 2M; en este sentido es necesario el entrenamiento de los recolectores en el método estándar para lograr mejoras en la productividad. 
DOI: http://dx.doi.org/10.22201/fi.25940732e.2017.18n2.011

Ocampo-lópez O.L., Ovalle-Castiblanco A.M., Arroyave-Diaz A., Salazar-Ospina K., Ramírez-Gómez C.A., Oliveros-Tascon C.E.,

Tabla 3. Indicadores de cosecha con el sistema tradicional y Canguaro $2 \mathrm{M}$

\begin{tabular}{|c|c|c|c|c|c|c|c|}
\hline $\begin{array}{c}\text { Indicador de } \\
\text { Cosecha }\end{array}$ & Variable & Método & Media & $\begin{array}{l}\text { Desviación } \\
\text { típica }\end{array}$ & $\begin{array}{l}\text { Error típ. de la } \\
\text { media }\end{array}$ & $\begin{array}{c}\text { Sig. } \\
\text { Prueba } \mathrm{T}\end{array}$ & $\begin{array}{l}\text { Sig. Prueba U } \\
\text { Mann- Whitney }\end{array}$ \\
\hline \multirow{2}{*}{ Eficiencia } & \multirow{2}{*}{ Rendimiento kg/h } & Tradicional & 10.43 & 2.96 & 0.43 & \multirow{2}{*}{0.057} & \multirow{2}{*}{0.076} \\
\hline & & Canguaro 2M & 9.23 & 2.52 & 0.37 & & \\
\hline \multirow[b]{2}{*}{ Eficacia } & \multirow{2}{*}{$\begin{array}{l}\text { Número de frutos } \\
\text { maduros no } \\
\text { recolectados por } \\
\text { árbol }\end{array}$} & Tradicional & 2.9 & 0.8 & 0.11 & \multirow[b]{2}{*}{$0.020^{*}$} & \multirow[b]{2}{*}{$0.038^{*}$} \\
\hline & & Canguaro $2 \mathrm{M}$ & 2.5 & 0.7 & 0.10 & & \\
\hline \multirow{4}{*}{ Calidad } & \multirow{2}{*}{$\begin{array}{l}\text { Porcentaje de } \\
\text { frutos maduros }\end{array}$} & Tradicional & 79.49 & 7.31 & 1.08 & \multirow{2}{*}{$0.040^{*}$} & \multirow{2}{*}{$0.042^{*}$} \\
\hline & & Canguaro $2 \mathrm{M}$ & 82.40 & 6.01 & 0.89 & & \\
\hline & \multirow{2}{*}{$\begin{array}{l}\text { Porcentaje de } \\
\text { frutos verdes }\end{array}$} & Tradicional & 0.78 & 0.49 & 0.07 & \multirow{2}{*}{$0.024^{*}$} & \multirow{2}{*}{$0.010^{*}$} \\
\hline & & Canguaro 2M & 0.55 & 0.48 & 0.07 & & \\
\hline \multirow{2}{*}{ Pérdidas } & \multirow{2}{*}{$\begin{array}{l}\text { Número de frutos } \\
\text { caídos al suelo } \\
\text { por árbol }\end{array}$} & Tradicional & 4.46 & 1.07 & 0.16 & \multirow{2}{*}{$0.000^{*}$} & \multirow{2}{*}{$0.000^{*}$} \\
\hline & & Canguaro 2M & 3.33 & 1.37 & 0.20 & & \\
\hline
\end{tabular}

Finalmente, la incorporación de herramientas de ingeniería industrial como el estudio de métodos en los procesos agrícolas permite su estandarización y brinda elementos a los caficultores para el entrenamiento de los nuevos recolectores. Por otra parte, facilita la identificación de aspectos de mejora y el control del proceso con el uso de indicadores de cosecha.

\section{Referencias}

Arcila J., Farfán F., Moreno A., Salazar L.F., Hincapié E. Sistemas de producción de café en Colombia, Federación Nacional de Cafeteros de Colombia, CENICAFÉ, Chinchiná, 2007, 309 p.

Arroyave A., Salazar K., Ocampo O.L., Ovalle A.M., Ramírez, C.A, Oliveros, C.E. Therbligs analysis of the coffee picking process with Canguaro 2M, en: 25 International conference on coffee science, ASIC, Armenia, Septiembre 8-13, 2014.

Bitzer V., Francken M., Glasbergen P. Alianzas Intersectoriales para una cadena de café sostenible: ¿Teniendo realmente en cuenta la sostenibilidad o solamente recogiendo las cerezas del café? Ensayos sobre economía cafetera, volumen 25, 2009: 123-145.

Cadena G. Ingeniería y Agro. Revista de Ingeniería, volumen 33, enero-junio, 2011: 70-87

Carreira X.C., Mariño R.A., Perez-Cancio X., Fernández M.E. Design of algorithms for evaluating the efficiency of labour and variability of some group milking parlour parameters. Livestock Science, volumen 151 (número 2), 2013: 252-263.

Durán F.A. Ingeniería de Métodos. Globalización: Técnicas para el manejo Eficiente de Recursos en Organizaciones fabriles, de Servicios y Hospitalarias, Guayaquil, Ecuador, 2007.

Fairtrade Foundation. Fairtrade and Coffee, Commodity Briefing, Londres, $25 \mathrm{p}$.
Farah A. Coffee Constituents, en: Yi-Fang. Emerging Health Effects and Disease Prevention, John Wiley \& Sons, 2012, pp. 21- 58.

Federación Nacional de Cafeteros de Colombia, CENICAFÉ. Manual del cafetero colombiano, Investigación y tecnología para la sostenibilidad de la caficultura, Tomo 3, 2013.

Federación Nacional de Cafeteros de Colombia. Informe del Gerente General 2015. LXXXII Congreso Nacional de Cafeteros, Bogotá, 2016, 53 p.

González-Pérez M.A. y Gutiérrez-Viana S. Cooperation in coffee markets: the case of Vietnam and Colombia. Journal of Agribusiness in Developing and Emerging Economies, volumen 2 (número 1), 2012: 57-73.

Isaza-Gil L.E., Montoya-Restrepo E.C., Vélez-Zape J.C., OliverosTascón, C.E. Evaluación de la concentración de los frutos maduros de café empleando técnicas no selectivas de recolección manual. Cenicafé, volumen 57 (número 4), 2006: 274-287.

Kanawaty G. Introducción al Estudio del Trabajo, 4 ed., Ginebra, Limusa, 2011.

López-Fisco H.A., Carlos E., Oliveros-Tascón C.E, Ramírez-Gómez C.A., Sanz-Uribe J.A. Avances técnicos. Manga para la recolección manual de café. Experiencia de investigación participativa, volumen 374, 2008b: 1-8.

López-Fisco H.A., Ramírez-Gómez C.A., Oliveros-Tascón C.E., Sanz-Uribe J.R. Aroandes, una tecnología para la cosecha manual de café con alta calidad. Cenicafé, volumen 590 (número 4), 2008a: 283-294.

Martínez-Recalde R., Montoya-Restrepo E., Vélez-Zape J., Oliveros-Tascón C.E. Estudio de tiempos y movimientos en la recolección manual de café en condiciones de alta pendiente. Cenicafé, volumen 56 (número 1), 2005: 50-66.

Meyers F.E. Estudios de tiempos y movimientos para la manufactura ágil, $2^{a}$ ed., México, Pearson Educación, 2000. 
DOI: http://dx.doi.org/10.22201/fi.25940732e.2017.18n2.011

NuEVo mÉtOdo ESTÁNDAR PARA LA RECOLECCIÓN SELECTIVA DE CAFÉ

Murthy P.S. y Naidu M. Sustainable management of coffee industry by-products and value addition -A review. Resources, Conservation and Recycling, volumen 66, 2012: 45-58.

Niebel B.W. y Freivalds A. Ingeniería Industrial. Métodos, estándares y diseño del trabajo, 11 a ed., Bogotá, Alfaomega, 2004.

Oliveros-Tascón C.E. y Sanz-Uribe J.R. Ingeniería y Café en Colombia. Revista de Ingeniería, Universidad de los Andes, volumen 33, 2011: 99-114.

Organización Internacional del Café. Anuario 2013-2014 fortaleciendo el sector cafetero mundial mediante la cooperación internacional, Londres, 2015, 36 p.

Organización Internacional del Café. Anuario 2014-2015 fortaleciendo el sector cafetero mundial mediante la cooperación internacional, Londres, 2016, $30 \mathrm{p}$.

Ovalle A.M. y Cárdenas D.M. ¿Qué ha pasado con la aplicación del estudio de tiempos y movimientos en las últimas dos décadas? Revisión de la literatura. Revista Ingeniería, Investigación y Desarrollo, volumen 16 (número 2), 2016:12-31.

Poveda G., Turbay S., Vélez J.J., Ocampo O.L., Acevedo E.C., Bedoya M. No sé qué vamos a hacer con estos climas! Vulnerabilidad y adaptación a las variaciones climáticas extremas en la cuenca de la quebrada Los Cuervos, afluente del río Chinchiná, Colombia. Medellín, Universidad de Antioquia, Universidad Nacional de Colombia, $33 \mathrm{p}$.

Quintero L. y Rosales M. El mercado mundial del café : tendencias recientes, estructura y estrategias de competitividad. Visión Gerencial, volumen 13 (número 2), 2014: 291-307.

Ramírez-Gómez C.A., Buenaventura J.D., Oliveros-Tascón C.E., Sanz-Uribe J.R. Informe final, cosecha manual asistida con Canguaro, Investigación Participativa ING-0175-IPA, Chinchiná, Caldas, Cenicafe, 2011.

Ramírez-Gómez C.A., Buenaventura J.D., Oliveros-Tascón C.E., Sanz-Uribe J.R. Equipo para la recolección manual de CaféCanguaro 2M. Experiencia de investigación participativa. Avances técnicos Cenicafé, volumen 438, 2013: 1-8.

Salazar K., Arroyave A, Ovalle A.M., Ocampo O.L., Ramírez C.A., Oliveros C.E. Tiempos en la recolección manual tradicional de café. Ingeniería Industrial, volume 37 (número 2), 2016: 114-126.

Salazar K., Arroyave A., Ocampo O.L., Ovalle A.M., Ramírez C.A, Oliveros C.E. Assessment of coffee picking process indicators, en: 25 International conference on coffee science, ASIC, Armenia, Septiembre 8-13, 2014.

Spinelli R. y Visser-Rien J.M. Analyzing and estimating delays in wood chipping operations. Biomass and Bioenergy, volumen 33 (número 3), 2009: 429-433.

Tinoco H.A., Ocampo D.A., Peña F.M., Sanz-Uribe J R. Finite element modal analysis of the fruit-peduncle of Coffea arabica L. var. Colombia estimating its geometrical and mechanical pro- perties. Computers and Electronics in Agriculture, volumen 108, 2014: 17-27.

Tinoco H.A., Ovalle A.M., Vargas C.A., Cardona M.J. An automated time and hand motion analysis based on planar motion capture extended to a virtual environment. Journal of Industrial Engineering International, volumen 11 (número 3), 2015: 391-402.

Torres P., Perez A., Marmolejo L.F., Ordoñez J.A., García R.E. Una mirada a la agroindustria de extracción de almidón de yuca, desde la estandarización de procesos. Revista Escuela de Ingeniería de Antioquia, volumen 14, 2010: 23-39.

Turbay S., Nates B., Jaramillo F., Vélez J.J., Ocampo O.L. Adaptación a la variabilidad climática entre los caficultores de las cuencas de los ríos Porce y Chinchiná, Colombia. Investigaciones Geográficas, Boletín del Instituto de Geografía, UNAM, volumen 85, 2014: 95-112.

Vélez-Zape J.C., Montoya E.C., Oliveros C.E. Estudio de tiempos y movimientos para el mejoramiento de la cosecha manual de café, Chinchiná, CENICAFÉ. Boletín Técnico 21. 1999, p.91

Villegas M.J., Montoya-Restrepo E.C., Vélez-Zape J.C., OliverosTascón C.E. Desempeño de los recolectores de café según la altura de la plantación. CENICAFÉ, volumen 56 (número 1), 2005: 19-36. 
DOI: http://dx.doi.org/10.22201/fi.25940732e.2017.18n2.011

Ocampo-lópez O.L., Ovalle-Castiblanco A.M., Arroyave-Diaz A., Salazar-Ospina K., Ramírez-Gómez C.A., Oliveros-Tascon C.E.,

\section{Citación sugerida:}

\section{Citación estilo Chicago}

Ocampo-López Olga Lucía, Alex Mauricio Ovalle-Castiblanco, Alejandro Arroyave-Diaz, Katherine Salazar-Ospina, Cesar Augusto Ramírez-Gómez, Carlos Eugenio Oliveros-Tascon. Nuevo método estándar para la recolección selectiva de café. Ingeniería Investigación y Tecnología, XVIII, 02 (2017): 127-137.

\section{Citación estilo ISO 690}

Ocampo-López O.L., Ovalle-Castiblanco A.M., Arroyave-Diaz A., Salazar-Ospina K., Ramírez-Gómez C.A., Oliveros-Tascon C.E. Nuevo método estándar para la recolección selectiva de café. Ingeniería Investigación y Tecnología, volumen XVIII (número 2), abril-junio 2017: 127-137.

\section{SemblanZas de los autores}

Olga Lucia Ocampo-López. Es ingeniera química por la Universidad Nacional de Colombia; asimismo es especialista en ciencia y tecnología de alimentos por la Universidad Nacional de Colombia sede Manizales y especialista en ingeniería ambiental en la misma universidad. Es magister en ingeniería química y candidata a doctor en ingeniería automática en la Universidad Nacional de Colombia sede Manizales. Es profesor asociado al programa de ingeniería industrial de la Universidad Autónoma de Manizales, grupo de investigación en diseño mecánico y desarrollo industrial reconocido por COLCIENCIAS.

Alex Mauricio Ovalle-Castiblanco. Ingeniero industrial, Universidad Nacional de Colombia, sede Manizales; es especialista en gerencia de negocios internacionales de la Universidad Jorge Tadeo Lozano de Bogotá-Colombia y magister en creatividad e innovación en las organizaciones de la Universidad Autónoma de Manizales-Colombia. Es profesor asociado al programa de ingeniería industrial de la Universidad Autónoma de Manizales, grupo de investigación en diseño mecánico y desarrollo industrial reconocido por COLCIENCIAS.

Alejandro Arroyave-Díaz. Ingeniero industrial por la Universidad Autónoma de Manizales, es investigador e innovador en el marco del convenio especial de cooperación suscrito entre la Universidad Autónoma de Manizales (UAM) y el Departamento Administrativo de Ciencia, Tecnología e Innovación, de Colombia (COLCIENCIAS).

Katherine Salazar-Ospina. Es ingeniera industrial por la Universidad Autónoma de Manizales, es investigadora e innovadora dentro del marco del convenio especial de cooperación suscrito entre la Universidad Autónoma de Manizales (UAM) y el Departamento Administrativo de Ciencia, Tecnología e Innovación, de Colombia (COLCIENCIAS).

César Augusto Ramírez-Gómez . Es arquitecto por la Universidad Nacional de Colombia, magister en desarrollo sostenible y medio ambiente por la Universidad de Manizales-Colombia e investigador científico I en ingeniería agrícola del Centro Nacional de Investigación del café (Cenicafè), Federación Nacional de Cafeteros de Colombia (FNC).

Carlos Eugenio Oliveros-Tascon. Es ingeniero agrícola por la Universidad del Valle-Colombia; magister en ingeniería agrícola por la Universidade Federal De Viçosa-Brasil y Phd. en ingeniería agrícola por la University of Wisconsin-US. Es investigador principal en ingeniería agrícola del Centro Nacional de Investigación del café (Cenicafè)- Federación Nacional de Cafeteros de Colombia (FNC). 